\title{
A Sensitive and Robust Ultra HPLC Assay with Tandem Mass Spectrometric Detection for the Quantitation of the PARP Inhibitor Olaparib (AZD2281) in Human Plasma for Pharmacokinetic Application
}

\author{
Jeffrey Roth ${ }^{1}$, Cody J. Peer ${ }^{1}$, Baskar Mannargudi ${ }^{1}$, Helen Swaisland ${ }^{2}$, Jung-Min Lee ${ }^{3}$, \\ Elise C. Kohn ${ }^{3}$ and William D. Figg ${ }^{1, *}$
}

1 Clinical Pharmacology Program, Office of the Clinical Director, National Cancer Institute, Bethesda, MD 20892, USA; E-Mails: jeffrey.roth@nih.gov (J.R.); cody.peer@nih.gov (C.J.P.); baskar.mm@gmail.com (B.M.)

2 AstraZeneca Pharmaceuticals, Alderley Park Macclesfield, Cheshire SK10 4TG, UK;

E-Mail: helen.swaisland@astrazeneca.com

3 Medical Oncology Branch, National Cancer Institute, Bethesda, MD 20892, USA;

E-Mails: leej6@mail.nih.gov (J.-M.L.); kohne@mail.nih.gov (E.C.K.)

* Author to whom correspondence should be addressed; E-Mail: figgw@helix.nih.gov;

Tel: +1-301-402-3622; Fax: +1-301-402-8606.

Received: 26 March 2014; in revised form: 12 May 2014 / Accepted: 12 June 2014 /

Published: 19 June 2014

\begin{abstract}
Olaparib (AZD2281) is an orally active PARP-1 inhibitor, primarily effective against cancers with BRCA1/2 mutations. It is currently in Phase III development and has previously been investigated in numerous clinical trials, both as a single agent and in combination with chemotherapy. Despite this widespread testing, there is only one published method that provides assay details and stability studies for olaparib alone. A more sensitive uHPLC-MS/MS method for the quantification of olaparib in human plasma was developed, increasing the range of quantification at both ends $(0.5-50,000 \mathrm{ng} / \mathrm{mL})$ compared to previously published methods $(10-5,000 \mathrm{ng} / \mathrm{mL})$. The wider range encompasses $\mathrm{C}_{\text {MAX }}$ levels produced by typical olaparib doses and permits better pharmacokinetic modeling of olaparib elimination. This assay also utilizes a shorter analytical runtime, allowing for more rapid quantification and reduced use of reagents. A liquid-liquid extraction was followed by chromatographic separation on a Waters UPLC ${ }^{\circledR}$ BEH C18 column $(2.1 \times 50 \mathrm{~mm}, 1.7 \mu \mathrm{m})$ and mass spectrometric detection. The mass transitions $m / z \quad 435.4 \rightarrow 281.1$ and $\mathrm{m} / \mathrm{z}$ $443.2 \rightarrow 281.1$ were used for olaparib and the internal standard $\left[{ }^{2} \mathrm{H}_{8}\right]$-olaparib, respectively.
\end{abstract}


The assay proved to be accurate $(<9 \%$ deviation) and precise $(\mathrm{CV}<11 \%)$. Stability studies showed that olaparib is stable at room temperature for $24 \mathrm{~h}$. in whole blood, at $4{ }^{\circ} \mathrm{C}$ for $24 \mathrm{~h}$ post-extraction, at $-80{ }^{\circ} \mathrm{C}$ in plasma for at least 19 months, and through three freeze-thaw cycles. This method proved to be robust for measuring olaparib levels in clinical samples from a Phase I trial.

Keywords: poly(ADP-ribose) polymerase inhibitor; olaparib; ultra-high performance liquid chromatography; tandem mass spectrometry; pharmacokinetics

\section{Introduction}

Olaparib (4-[[3-[4-(cyclopropanecarbonyl)piperazine-1-carbonyl]-4-fluorophenyl]methyl]-2H-phthalazin1-one; AZD2281) is an orally active inhibitor of poly(ADP-ribose) polymerases (PARP), of which PARP-1 is the most common. This enzyme assists with base-excision repair, fixing single-strand DNA breaks [1] that, if not repaired, become double-strand breaks when encountered by the replication fork. Double-strand breaks are often repaired via homologous recombination (HR), where BRCA1 and BRCA2 play an important role [2]. Deleterious mutations in BRCA1 or BRCA2 predispose carriers to breast and other cancers. When PARP is inhibited, BRCA1/2-deficient cells can no longer efficiently repair double-strand DNA breaks, leading to accumulation of DNA lesions, chromatid breaks, and eventual apoptosis. Thus, tumor cells with BRCA1/2 mutations are potentially sensitized to PARP inhibitors. Murai et al. reported that, in addition to inhibiting base-excision repair, PARP inhibitors exhibit further cytotoxic activity by trapping PARP at single-strand DNA breaks [3]. The mechanism of PARP inhibitors offer the possibility of selectively killing cancer cells while avoiding the toxicities associated with most chemotherapy.

Farmer et al. found that mouse embryonic stem cells lacking BRCA1 and BRCA2 are 1000 times more sensitive to PARP inhibitors than wild-type cells [2]. An early phase I trial of the PARP inhibitor olaparib documented antitumor activity in carriers of BRCA mutations [4]. Since then, olaparib has shown clinical efficacy, either through clinical responses or pharmacodynamics endpoints (biomarkers), against BRCA-mutant breast [5-7] and ovarian [8-12] cancers and has also shown efficacy in less common BRCA-related cancers, such as lung, uterine, pancreatic, mesothelioma, kidney, and prostate cancer [4]. Olaparib has also been used to enhance tumor sensitivity to camptothecin and radiation [13,14].

Several clinical trials have quantitatively measured olaparib in plasma for pharmacokinetic analyses, either as single-agent therapy [4,5,15] or in combination with such drugs as dacarbazine [16], topotecan [17], bevacizumab [18], or cisplatin and gemcitabine [19]. However, the method details of those assays and stability studies were not published. The only published analytical assay that contains useful extraction, stability, and methodology details for single-agent olaparib was calibrated over the range of $10-5,000 \mathrm{ng} / \mathrm{mL}$ [20]. A common olaparib dose is $300 \mathrm{mg}$ (tablet) or $400 \mathrm{mg}$ (capsule) twice daily, which is well absorbed and tends to produce $\mathrm{C}_{\mathrm{MAX}}$ levels around or above the upper calibration limit of 5,000 $\mathrm{ng} / \mathrm{mL}$. Thus, an upper limit of quantification (ULOQ) greater than $5,000 \mathrm{ng} / \mathrm{mL}$ in the calibration curve, or a validated dilution to within the calibration range that allows for analysis of plasma concentrations above the ULOQ, is needed to properly and accurately quantitate olaparib at 
commonly encountered high plasma levels during pharmacokinetic studies. A previous study seemed to indicate that diluting samples with olaparib concentrations above the ULOQ was feasible, but did not fully validate this technique [20].

In order to most accurately determine olaparib plasma concentrations around the highest concentrations ( $\sim 0.5-3 \mathrm{~h}$ post dose), wide ranging steady-state peak and trough concentrations (during repeated dosing every $12 \mathrm{~h}$ ), and the lowest concentrations (during elimination after the final dose), an analytical assay must have a wide calibration range with excellent sensitivity and the capability of diluting very high concentrations above the calibration range. There is a need for a robust analytical assay for olaparib alone that is accurate and precise over a wide range for use in pharmacokinetic analyses on clinical studies. Patients taking olaparib commonly experience peak concentrations shortly after dosing, wide ranging steady-state concentrations, and low concentrations during the terminal elimination phase. Measuring low levels of olaparib following the last dose allows for proper pharmacokinetic determination of half-life and clearance values. Described here is a simple and reliable ultra HPLC assay with tandem mass spectrometric (uHPLC-MS/MS) detection for olaparib in human plasma from concentrations encompassing $0.5-50,000 \mathrm{ng} / \mathrm{mL}$.

\section{Experimental Section}

\subsection{Materials}

Olaparib and $\left[{ }^{2} \mathrm{H}_{8}\right]$-olaparib were provided by AstraZeneca (Waltham, MA, USA). The chemical structures of olaparib and $\left[{ }^{2} \mathrm{H}_{8}\right]$-olaparib are shown in Figure 1. Optima-grade acetonitrile, methanol, and ethyl acetate were obtained from Fisher Scientific (Fairlawn, NJ, USA) and de-ionized water was generated with a Hydro-Reverse Osmosis system (Durham, NC, USA) connected to a Milli-Q UV Plus purifying system (Billerica, MA, USA). Drug-free human heparinized plasma was obtained from the NIH Clinical Center Blood Bank (Bethesda, MD, USA).

Figure 1. Structures of olaparib (A) and the internal standard ${ }^{2}\left[\mathrm{H}_{8}\right]$-olaparib (B).

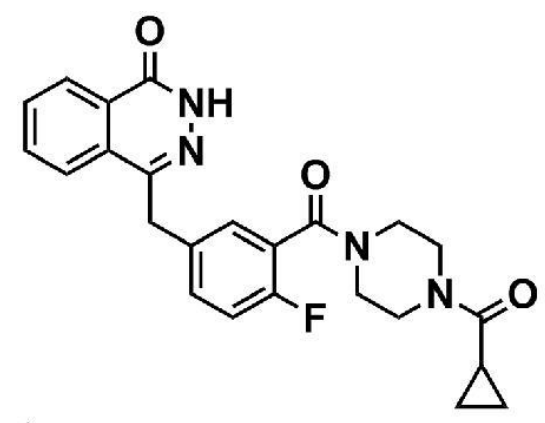

A.

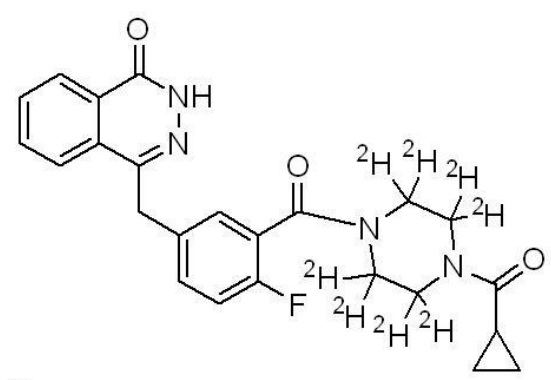

B

\subsection{Preparation of Stock Solutions}

Master stock solutions were prepared individually by dissolving olaparib and $\left[{ }^{2} \mathrm{H}_{8}\right]$-olaparib in methanol at free base concentrations of $1 \mathrm{mg} / \mathrm{mL}$. After vortex mixing and brief sonication, each stock solution was stored in glass vials at $-80{ }^{\circ} \mathrm{C}$. Serial dilutions (working stocks) were prepared in 
acetonitrile from each individual master stock and stored in glass vials at $-80{ }^{\circ} \mathrm{C}$ for the preparation of calibration and quality control (QC) samples.

For each analytical run, calibration standards in drug-free human heparinized plasma were freshly prepared in duplicate at final plasma concentrations of $0.5,5,25,100,500,1,000,2,000$, and 5,000 ng/mL. Quality control (QC) samples were prepared in batch at concentrations of 1.0 (low-range; LQC), 900 (mid-range; MQC), 4,500 (high-range; HQC), and 50,000 ng/mL (10-fold dilution; DQC) by adding drug-free heparinized plasma to the required amount of working stock in a volumetric flask. QC samples were vortex-mixed, then subdivided into aliquots and stored at $-80{ }^{\circ} \mathrm{C}$.

\subsection{Sample Preparation}

Frozen standards and samples were thawed on wet ice before homogenization by vortex-mixing. A liquid-liquid extraction was performed by adding $1 \mathrm{~mL}$ of ethyl acetate containing $10 \mathrm{ng} / \mathrm{mL}$ $\left[{ }^{2} \mathrm{H}_{8}\right]$-olaparib (internal standard) to $100 \mu \mathrm{L}$ of plasma calibrator aliquots, QC samples, and study samples. This mixture was briefly vortexed, then centrifuged for $10 \mathrm{~min}$ at $13,200 \mathrm{rpm}(11,700 \times g)$ in a mini-centrifuge before the supernatant was dried down and reconstituted with $100 \mu \mathrm{L}$ water/acetonitrile, 50/50.

\subsection{Instrument Conditions}

Ten microliters of each sample was injected onto a Waters ACQUITY UPLC ${ }^{\circledR}$ system (Waters Corporation, Milford, MA, USA), which included a binary pump, refrigerated autosampler $\left(4^{\circ} \mathrm{C}\right)$ and a temperature-controlled column compartment $\left(30^{\circ} \mathrm{C}\right)$. Chromatographic separation was achieved on a Waters UPLC ${ }^{\circledR}$ BEH C18, $2.1 \times 50$ mm, $1.7 \mu \mathrm{m}$ preceded by a Waters UPLC ${ }^{\circledR}$ BEH C18 guard column packed with $1.7-\mu \mathrm{m}$ packing material. The mobile phase consisted of A: $0.1 \%$ formic acid in water, and B: $0.1 \%$ formic acid in acetonitrile with a flow rate of $0.25 \mathrm{~mL} / \mathrm{min}$ and a total run time of $5 \mathrm{~min}$. A gradient of mobile phase B was utilized by increasing the initial $20 \%$ linearly to $80 \%$ over 2 min, up to $100 \%$ by $2.1 \mathrm{~min}$ and held there until $3.1 \mathrm{~min}$, before returning to $20 \%$ by $3.2 \mathrm{~min}$. The column eluent was directed into a Waters Quattro Micro triple quadrupole mass spectrometer (Waters, Milford, MA, USA). The mass spectrometer was set to monitor olaparib $(\mathrm{m} / z \quad 435.4 \rightarrow 281.1)$ and the internal standard $\left[{ }^{2} \mathrm{H}_{8}\right]$-olaparib $(\mathrm{m} / \mathrm{z} 443.2 \rightarrow 281.1)$ using multiple reaction monitoring (MRM) in the positive ion mode. The fragmentation pattern of olaparib was previously described [21]. Universal mass spectrometric settings included capillary voltage of $4.0 \mathrm{kV}$, cone voltage of $30 \mathrm{~V}$, extractor voltage 3.0 V, RF Lens 1.5 , source temperature of $110{ }^{\circ} \mathrm{C}$, desolvation temperature $450{ }^{\circ} \mathrm{C}$, cone gas flow $50 \mathrm{~L} / \mathrm{h}$, desolvation gas flow $700 \mathrm{~L} / \mathrm{h}$, collision energy of 20 , and dwell times of $200 \mathrm{msec}$. MRM peak integrations and data analyses were performed using the QuanLynx program in MassLynx 4.1 (Waters Corp, Milford, MA, USA).

\subsection{Validation}

\subsubsection{Linearity}

Calibration curves for olaparib were constructed by least-squares linear regression analysis of an eight-point calibration curve $(0.5-5,000 \mathrm{ng} / \mathrm{mL})$ by plotting the ratio of the analyte peak area versus 
the internal standard peak area, using $1 / x^{2}$ as a weighting factor, where $x$ is the ratio of the nominal analyte: IS concentration. Calibrator response functions and choice of regression analysis were investigated by calculating correlation coefficients $\left(r^{2}\right)$ and the percent deviation $(\% \mathrm{DEV})$ for all calibrators.

\subsubsection{Accuracy and Precision}

Accuracy and precision were evaluated by determining olaparib at four different concentrations of QC samples (low-range, mid-range, high-range, 10-fold dilution), in five replicates analyzed over four different days. The DQC was prepared by 10 -fold dilution of a $50,000 \mathrm{ng} / \mathrm{mL}$ plasma solution with drug-free plasma to bring it within the limits of the calibration curve. The LLOQ was analyzed in five different lots of plasma. Each run consisted of blank plasma extracts, internal standard only extracts, calibration standards in duplicate, and QC samples in replicates of five, each of the four days $(n=20)$. Accuracy $(\% \mathrm{DEV})$ was defined as the percent difference between the mean observed concentration and the nominal concentration. The repeatability of the assay was determined by the within-run precision (WRP) and between-run (BRP), as calculated below.

$$
\begin{aligned}
& \mathrm{WRP}=100 \times\left[\left(\mathrm{MS}_{\mathrm{WIT}}\right)^{\wedge 0.5} / \mathrm{GM}\right] \\
& \mathrm{BRP}=100 \times\left[\left(\left\{\mathrm{MS}_{\mathrm{BET}}-\mathrm{MS}_{\mathrm{WIT}}\right\} / \mathrm{n}\right)^{\wedge 0.5} / \mathrm{GM}\right]
\end{aligned}
$$

$\mathrm{GM}$ represents the grand mean, $\mathrm{MS}_{\mathrm{wit}}$ represents within-group mean squared, $\mathrm{MS}_{\text {bet }}$ represents between-group mean squared, and $n$ represents the number of repetitions. FDA guidelines for bioanalytical validation were followed, with $\pm 15 \%$ variability in accuracy and precision allowed, except for the LLOQ that is allowed $\pm 20 \%$ [22].

\subsubsection{Stability}

Olaparib has been previously shown to demonstrate plasma stability for $24 \mathrm{~h}$ at room temperature and $4{ }^{\circ} \mathrm{C}$ [21], but stability in whole blood can sometimes be different. This has importance in clinical studies, as blood samples are not always immediately centrifuged to plasma following blood draw into tubes with anti-coagulant. The stability of olaparib in whole blood at room temperature was assessed over a $24-\mathrm{h}$ period. Samples at two concentrations (3.75 and 3,750 $\mathrm{ng} / \mathrm{mL}$ ) were either extracted immediately (fresh) or let sit at room temperature in blood for $24 \mathrm{~h}$ before centrifugation to plasma and subsequent extraction, each in triplicate. The analyte concentration after $24 \mathrm{~h}$ was compared to the concentration of freshly prepared samples in the same analytical run.

Additional stability tests were performed to verify the absence of olaparib degradation in plasma during freeze/thaw cycles as previously demonstrated [21]. Samples were assayed at three concentrations $(6,150$, and $1,600 \mathrm{ng} / \mathrm{mL})$ in triplicate. The samples were subjected to three freeze/thaw cycles at $-80{ }^{\circ} \mathrm{C}$, with each freeze cycle lasting at least $12 \mathrm{~h}$. The analyte concentration after each storage period was compared to the concentration of freshly prepared samples in the same analytical run.

The post-preparative stability of olaparib and $\left[{ }^{2} \mathrm{H}_{8}\right]$-olaparib pending analysis in the $4{ }^{\circ} \mathrm{C}$ autosampler was assessed. Samples were re-injected and re-analyzed $24 \mathrm{~h}$ after the initial analysis and compared to the original values obtained from those same samples prepared $24 \mathrm{~h}$ prior.

Long-term stability of olaparib in plasma was tested by re-analyzing clinical samples drawn 19 months earlier. These samples had been stored at $-80{ }^{\circ} \mathrm{C}$ since the initial analysis. 


\subsubsection{Extraction Recovery and Matrix Effects}

The extraction efficiency of the liquid-liquid extraction was assessed by comparing analyte peak areas from both a low $(5 \mathrm{ng} / \mathrm{mL} ; n=5)$ and high $(2,000 \mathrm{ng} / \mathrm{mL} ; n=5)$ concentration, both before and after the addition of the liquid extraction solvent. For each set, both concentrations (low and high) were assayed in quintuplet by spiking analyte into five different lots of plasma to further demonstrate selectivity and matrix effects.

Matrix effects from the plasma on the mass spectrometric signals for olaparib and the internal standard $\left[{ }^{2} \mathrm{H}_{8}\right]$-olaparib were assessed through direct comparison of samples spiked in plasma to samples spiked in clean solution (reconstitution solution). Mass spectrometric peak areas for olaparib were first analyzed using a low $(5 \mathrm{ng} / \mathrm{mL} ; n=3)$ and a high $(2,000 \mathrm{ng} / \mathrm{mL} ; n=3)$ calibrator sample spiked into the reconstitution solution $\left(\mathrm{ACN} / \mathrm{H}_{2} \mathrm{O}, 50 / 50\right)$. These values were used for comparison to the same concentrations by spiking drug into each of 5 plasma lots after extraction (to avoid discrepancies from extraction efficiency).

\subsection{Clinical Application}

To demonstrate the applicability of this method for measuring a wide range of plasma olaparib concentrations clinically, blood samples were drawn just prior to a $200 \mathrm{mg}$ oral dose, as well as $0.5,1$, 2, 3, 4, 8, and $10 \mathrm{~h}$ post dose (NCT01237067). Blood was drawn into tubes containing sodium heparin and immediately placed on wet ice. The blood was then centrifuged to obtain plasma within $24 \mathrm{~h}$ of blood draw (typically $3 \mathrm{~h}$ post draw), which was then frozen until extraction and analysis by this assay. The Institutional Review Board of the National Cancer Institute approved this clinical protocol.

\section{Results and Discussion}

\subsection{Selectivity}

Figure 2 depicts LC-MS/MS chromatograms of a drug-free plasma extract (Figure 2a), the internal standard only (Figure 2b), the LLOQ at $0.5 \mathrm{ng} / \mathrm{mL}$ (Figure 2c), and a clinical pharmacokinetic sample taken $30 \mathrm{~min}$ following a single $200 \mathrm{mg}$ oral dose (Figure 2d). The retention time of both olaparib and its deuterated analog, $\left[{ }^{2} \mathrm{H}_{8}\right]$-olaparib was approximately $1.37 \mathrm{~min}$. The LLOQ had a signal to noise $(\mathrm{S} / \mathrm{N})$ ratio of 8.95 , which is well above the commonly accepted $\mathrm{S} / \mathrm{N}$ of 5 for LLOQ peaks. The use of ultra-high performance liquid chromatography (uHPLC) provided strong, sharp peaks that were well-resolved and separated from interfering matrix peaks that were eluted following an organic solvent gradient to cleanse the column. 
Figure 2. Chromatograms of (a) a blank plasma extract; (b) internal standard only extract; (c) the lower limit of quantification (LLOQ); and (d) a clinical pharmacokinetic sample drawn 30 min following a $200 \mathrm{mg}$ oral dose. In each pane, olaparib is on the bottom, the IS on top.
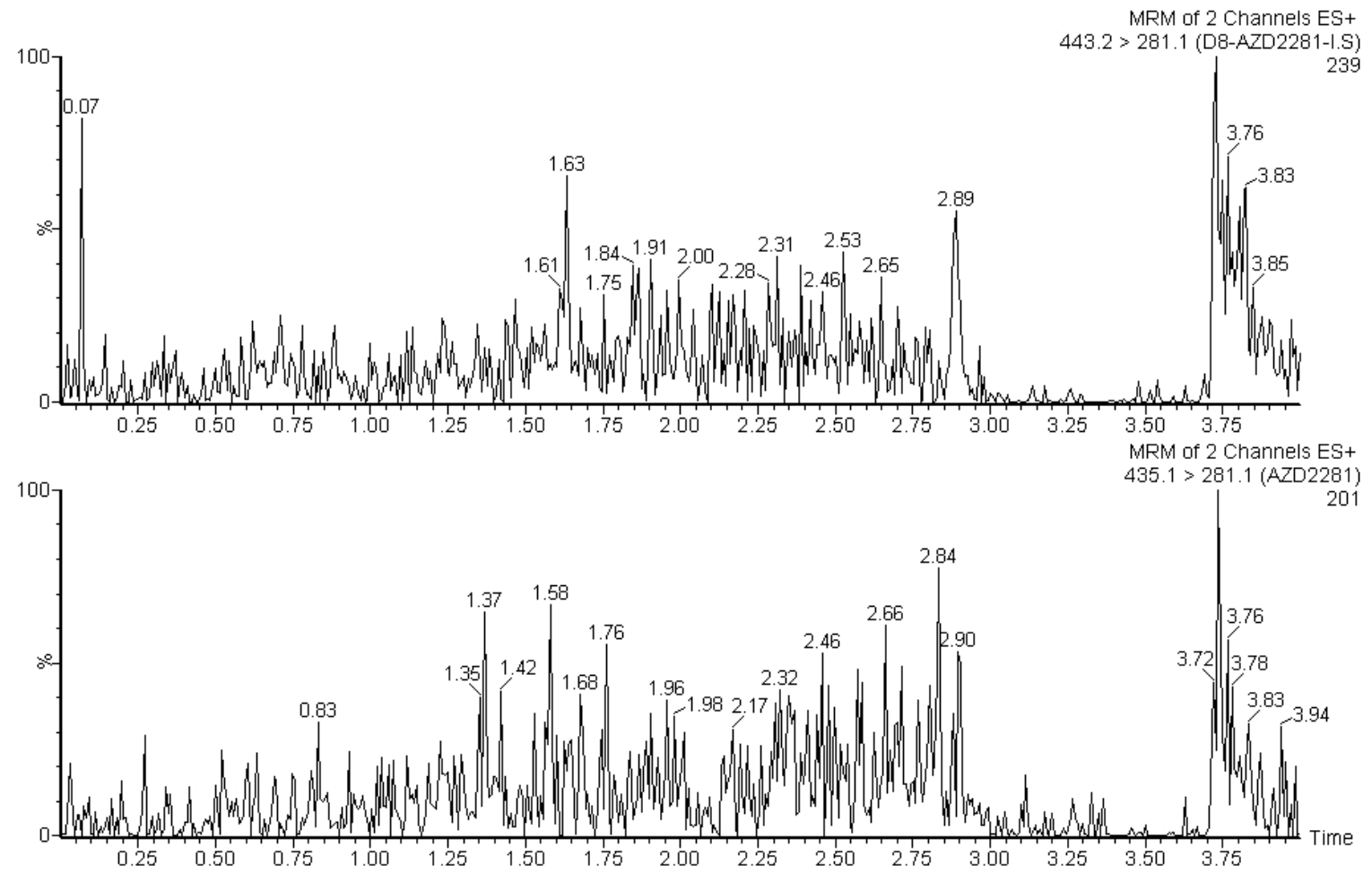

(a)
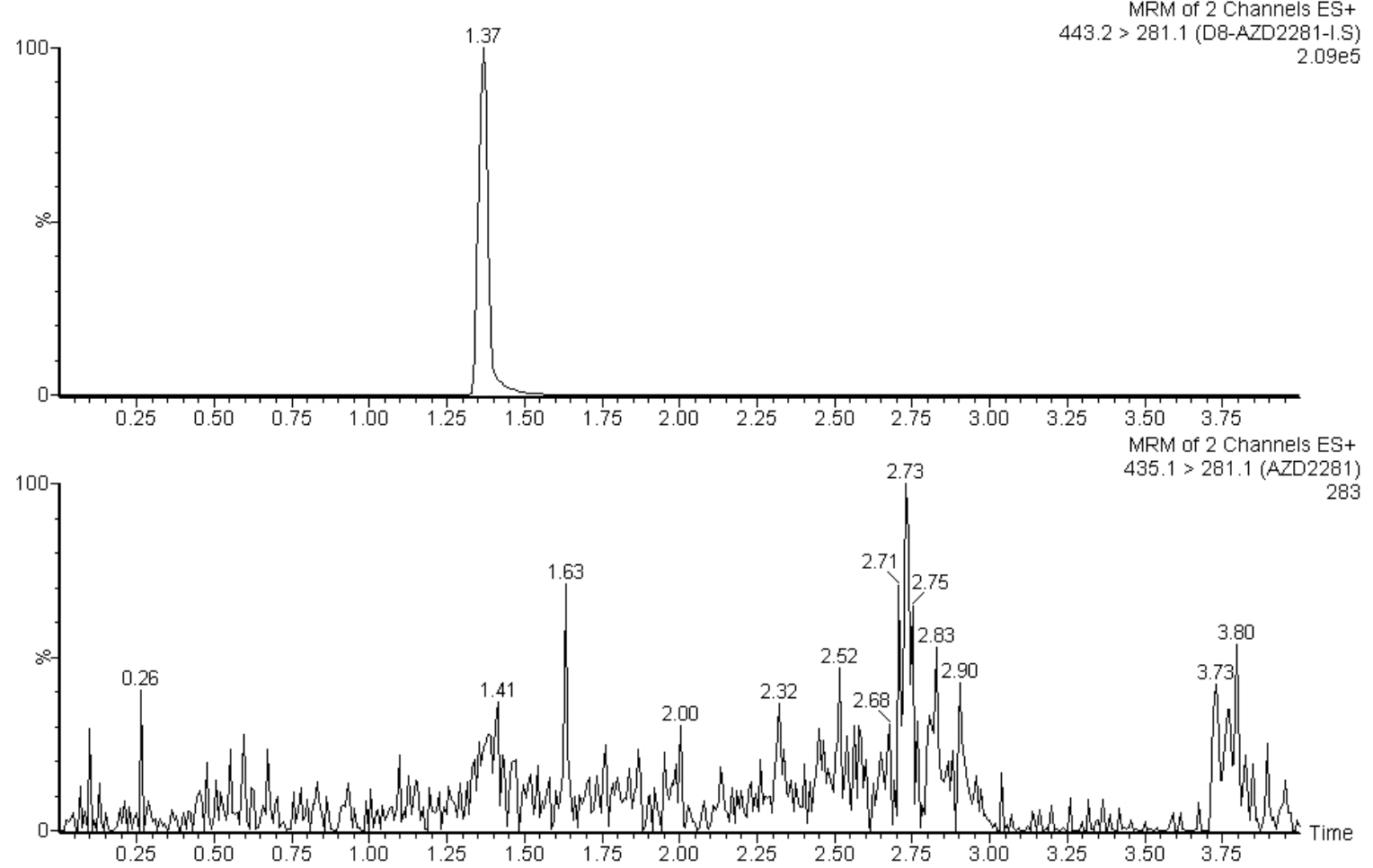

(b) 
Figure 2. Cont.
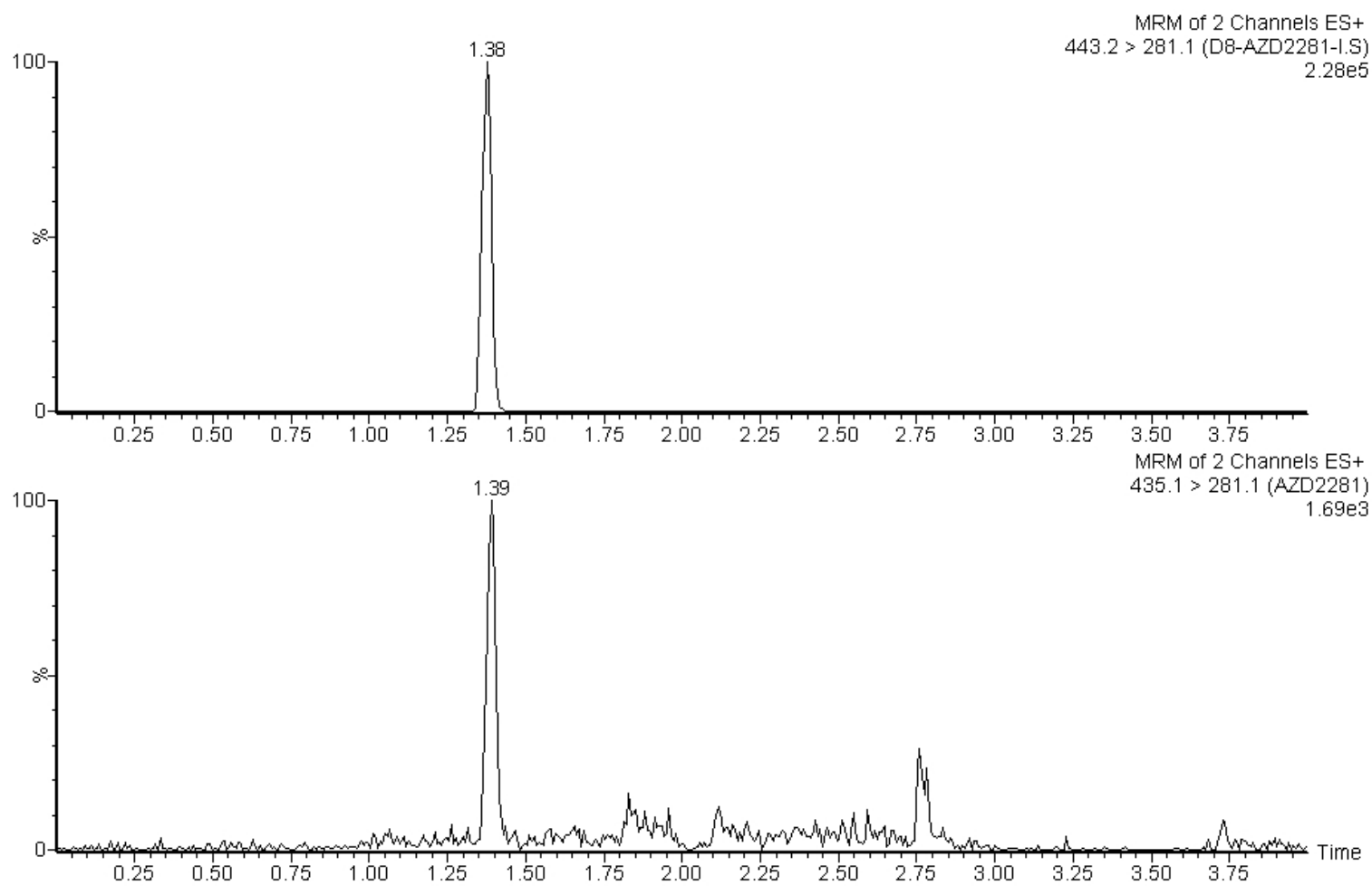

(c)
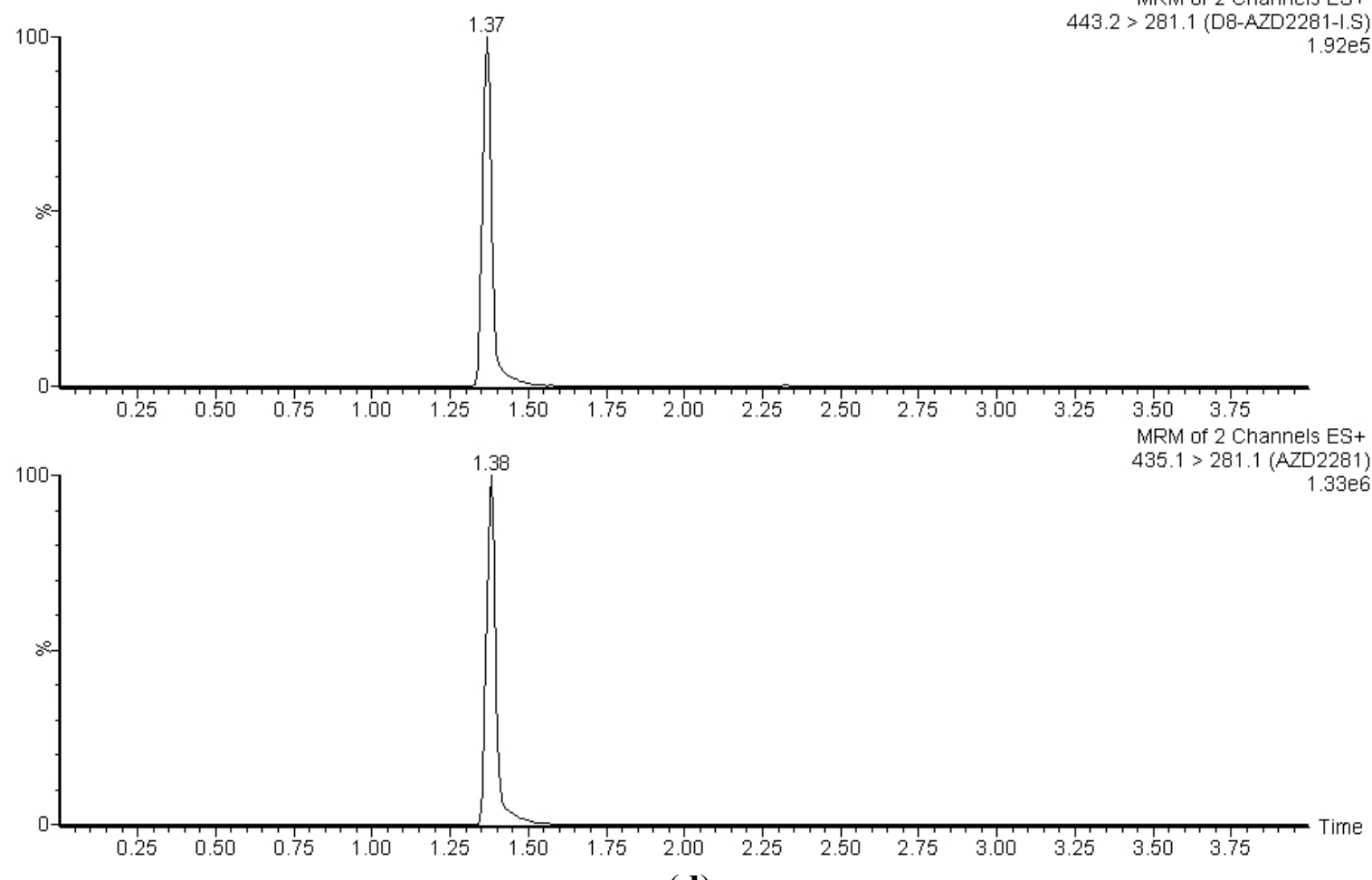

(d)

\subsection{Linearity, Accuracy and Precision}

The calibration standards, ranging from $0.5-5000 \mathrm{ng} / \mathrm{mL}$, were run in duplicate on each of four days $(n=8)$. The eight calibration standards proved accurate and precise (Table 1$)$, with a mean $( \pm \mathrm{SD})$ linear calibration curve correlation $\left(r^{2}\right)$ of $0.9958 \pm 0.0019(n=4)$. Quality control (QC) samples, at low 
(1 ng/mL), mid (900 ng/mL), high (4,500 ng/mL), and 10-fold diluted (50,000 ng/mL) concentrations were run in quintuplet daily over four days $(n=20)$. All QC levels proved accurate and precise, within the required $\pm 15 \%$ (Table 2 ).

Table 1. Calibration Linearity.

\begin{tabular}{cccccc}
\hline Nominal $(\mathbf{n g} / \mathbf{m L})$ & GM (ng/mL) & SD $(\mathbf{n g} / \mathbf{m L})$ & DEV (\%) & CV (\%) & $\boldsymbol{n}$ \\
\hline 0.50 & 0.52 & 0.06 & 3.00 & 11.88 & 8 \\
5.0 & 5.38 & 0.31 & 7.50 & 5.78 & 8 \\
25 & 24.99 & 1.17 & -0.04 & 4.67 & 8 \\
100 & 104.30 & 2.58 & 4.30 & 2.47 & 8 \\
500 & 494.13 & 16.09 & -1.17 & 3.26 & 8 \\
1000 & 1001.39 & 36.39 & 0.14 & 3.63 & 8 \\
2000 & 1963.61 & 74.09 & -1.82 & 3.77 & 8 \\
5000 & 4594.08 & 94.75 & -8.12 & 2.06 & 8 \\
\hline
\end{tabular}

Abbreviations: GM, grand mean; SD, standard deviation; DEV (\%), relative deviation from nominal value; $\mathrm{CV}(\%)$, coefficient of variation; $n$, number of replicate observations within each validation run, i.e., two samples at each concentration were run on four separate occasions, for a total $(n)$ of eight samples at each concentration.

Table 2. Accuracy and Precision.

\begin{tabular}{ccccccc}
\hline Nominal (ng/mL) & GM (ng/mL) & SD (ng/mL) & DEV (\%) & WRP (\%) & BRP (\%) & $\boldsymbol{n}$ \\
\hline 0.5 (LLOQ) & 0.49 & 0.05 & -2.60 & 9.10 & 1.75 & 20 \\
1.0 (LQC) & 1.01 & 0.12 & 1.10 & 10.79 & 3.65 & 20 \\
900 (MQC) & 876.89 & 39.72 & -2.57 & 1.53 & 2.77 & 20 \\
4500 (HQC) & 4105.04 & 133.88 & -8.78 & 1.59 & 1.85 & 20 \\
50,000 (DQC) & $48,028.85$ & 1397.15 & -3.94 & 2.20 & 1.24 & 20 \\
\hline
\end{tabular}

Abbreviations: GM, grand mean; SD, standard deviation; DEV (\%) relative deviation from nominal value; WRP, within-run precision; $n$, number of replicate observations within each validation run; BRP, between-run precision (A hyphen indicates $\mathrm{MS}_{\mathrm{wit}}>\mathrm{MS}_{\text {bet}}$, thus BRP cannot be calculated and it was concluded that no additional variation was observed as a result of performing the assay in different runs).

\subsection{Stability}

Olaparib demonstrated excellent bench-top stability in blood at room temperature through $24 \mathrm{~h}$, with $<1.50 \%$ difference in measured concentrations from freshly extracted samples at either a low or high concentration. Freeze/thaw stability was demonstrated through three cycles in human plasma, where changes in measured values at each of the three concentrations after each freeze/thaw cycle were below $10 \%$, indicating no significant degradation (Table 3), which is consistent with a previous report [21]. The 24-h post-preparative stability measurements following a liquid-liquid extraction were consistent with the initial run at each of the three concentrations tested ( $<1.00 \%$ change), allowing samples extracted from human plasma to be reanalyzed on the following day when necessary without significant degradation. Olaparib was shown to be stable in plasma at $-80{ }^{\circ} \mathrm{C}$ for at least 19 months (8 months longer than previously reported, when stored at $-20^{\circ} \mathrm{C}$ ) [20]. 
Table 3. Freeze/Thaw Stability in Plasma.

\begin{tabular}{|c|c|c|c|c|c|c|}
\hline & \multicolumn{2}{|c|}{$6.50 \mathrm{ng} / \mathrm{mL}$ (low) } & \multicolumn{2}{|c|}{$150 \mathrm{ng} / \mathrm{mL}$ (mid) } & \multicolumn{2}{|c|}{1600 (high) } \\
\hline $\begin{array}{c}\text { Freeze/Thaw } \\
\text { Cycles }\end{array}$ & $\begin{array}{c}\text { GM } \\
(\mathrm{ng} / \mathrm{mL})\end{array}$ & $\begin{array}{l}\text { DEV from } \\
\text { Fresh (\%) }\end{array}$ & $\begin{array}{c}\text { GM } \\
(\mathrm{ng} / \mathrm{mL})\end{array}$ & $\begin{array}{l}\text { DEV from } \\
\text { Fresh (\%) }\end{array}$ & $\begin{array}{c}\mathbf{G M} \\
(\mathbf{n g} / \mathbf{m L}) \\
\end{array}$ & $\begin{array}{l}\text { DEV from } \\
\text { Fresh (\%) }\end{array}$ \\
\hline 0 (Fresh) & 5.94 & - & 149.52 & - & 1489.40 & - \\
\hline 1 & 6.10 & 2.69 & 146.70 & -1.89 & 1512.20 & 1.53 \\
\hline 2 & 6.22 & 4.71 & 156.40 & 4.60 & 1606.48 & 7.86 \\
\hline 3 & 6.50 & 9.43 & 153.66 & 2.77 & 1572.46 & 5.58 \\
\hline
\end{tabular}

Abbreviations: GM, grand mean; DEV (\%) relative deviation from nominal value.

\subsection{Extraction Recovery and Matrix Effects}

The mean extraction efficiencies for olaparib, estimated by comparing the mass spectrometric signal response of the analyte spiked into 5 different lots of human plasma before extraction versus analyte spiked after extraction at both a low $(5 \mathrm{ng} / \mathrm{mL} ; n=5)$ and a high $(2,000 \mathrm{ng} / \mathrm{mL} ; n=5)$ concentration, were $70.97 \%$ and $87.35 \%$, respectively (previous method did not report percent recovery). Comparison of analyte peak areas between post-extraction plasma and clean solvent resulted in a $-40.21 \%$ and $-34.95 \%$ change in signal from plasma matrix effects for the low and high concentrations, respectively, but this did not affect linearity of the calibration.

\subsection{Clinical Application}

The mean olaparib plasma concentration $v s$. time curve for subjects $(n=8)$ receiving a $200 \mathrm{mg}$ tablet oral dose of olaparib was plotted (Figure 3 ). The maximum observed plasma concentration $\left(\mathrm{C}_{\mathrm{MAX}}\right)$ was approximately $10,000 \mathrm{ng} / \mathrm{mL}$ and occurred around $1 \mathrm{~h}$ post oral dose, suggesting rapid and extensive absorption and high bioavailability. In addition to the $\mathrm{C}_{\mathrm{MAX}}$, the sampling times just before and after were also above the ULOQ of $5000 \mathrm{ng} / \mathrm{mL}$, justifying the need for a validated dilution. Although plasma concentrations $10 \mathrm{~h}$ post-dose were still around $1,000 \mathrm{ng} / \mathrm{mL}$, the lower limit of quantification of $0.5 \mathrm{ng} / \mathrm{mL}$ is useful to best characterize elimination through the ability to accurately measure plasma concentrations up to $90 \mathrm{~h}$ post dose, based on a half-life of approximately $8 \mathrm{~h}$ [15].

Figure 3. Olaparib plasma concentration vs. time curve.

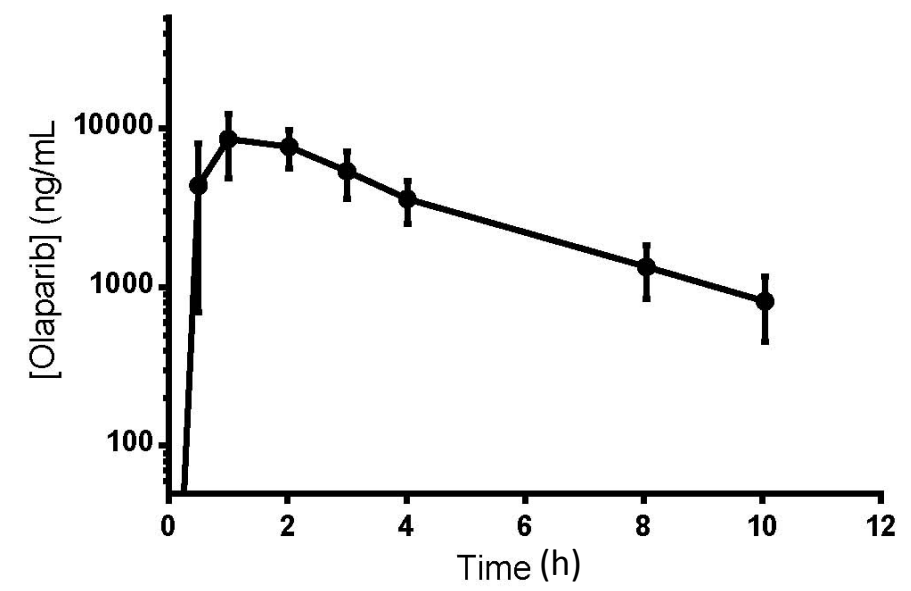




\section{Conclusions}

This paper describes a rapid, more sensitive analytical assay to singularly detect and accurately measure olaparib, a PARP-1 inhibitor, currently undergoing Phase III clinical testing in numerous trials. Previous literature assays for olaparib included other compounds or failed to encompass all clinically-relevant plasma concentrations. This method demonstrated selectivity, accuracy, precision, and efficiency at recovering drug (87\%) from plasma. Olaparib was found to be stable in plasma through three freeze/thaw cycles, at $-80{ }^{\circ} \mathrm{C}$ for at least 19 months, at room temperature for $24 \mathrm{~h}$ in whole blood, and demonstrated $24 \mathrm{~h}$ post-preparative stability at $4{ }^{\circ} \mathrm{C}$. The method's shorter runtime ( $5 \mathrm{~min}$ ) permits quicker analysis and conserves reagents. Sensitivity was improved over existing published methods by at least 20-fold (LLOQ $0.5 \mathrm{ng} / \mathrm{mL}$ ), which allows for better characterization of pharmacokinetic profiles to allow proper determination of half-life and clearance. The validated 10 -fold dilution, capable of measuring plasma levels up to $50,000 \mathrm{ng} / \mathrm{mL}$, allows for accurate and reliable measurements of peak concentrations above the ULOQ during high dose olaparib combined with other agents that may increase olaparib exposure, either through increased absorption or decreased clearance.

\section{Acknowledgments}

This study was funded in part by the Intramural Research Program of the NIH, National Cancer Institute and in part by an umbrella CRADA between the National Cancer Institute Center for Cancer Research (NCI/CCR) and AstraZeneca (2299). This is a US Government work. There are no restrictions on its use. The views expressed within this paper do not necessarily reflect those of the US Government. We thank the nursing staff of the National Cancer Institute and the fellows of the Medical Oncology Branch at the National Cancer Institute for their care of our patients. Most importantly, we appreciate the patients with cancer who enroll in investigational trials to advance the knowledge of this disease.

\section{Authors Contributions}

Jeffrey Roth: data generation, data analysis, manuscript writing and editing; Cody J. Peer: data generation, data analysis, conceptual design, manuscript writing and editing; Baskar Mannargudi: data generation, data analysis, conceptual design, manuscript editing; Helen Swaisland: manuscript editing; Jung-min Lee: clinical trial design and execution, manuscript editing; Elise C. Kohn: clinical trial design and execution, manuscript editing; William D. Figg: data analysis, manuscript writing and editing.

\section{Disclaimer}

The content of this publication does not necessarily reflect the views or policies of the Department of Health and Human Services, nor does mention of trade names, commercial products, or organization imply endorsement by the U.S. Government. The views in this manuscript are those of the authors and may not necessarily reflect NIH policy. No official endorsement is intended nor should be inferred. 


\section{Conflicts of Interest}

The authors declare no conflict of interest.

\section{References}

1. Dantzer, F.; de La Rubia, G.; Menissier-de Murcia, J.; Hostomsky, Z.; de Murcia, G.; Schreiber, V. Base excision repair is impaired in mammalian cells lacking Poly(ADP-ribose) polymerase-1. Biochemistry 2000, 39, 7559-7569.

2. Farmer, H.; McCabe, N.; Lord, C.J.; Tutt, A.N.; Johnson, D.A.; Richardson, T.B.; Santarosa, M.; Dillon, K.J.; Hickson, I.; Knights, C.; et al. Targeting the DNA repair defect in BRCA mutant cells as a therapeutic strategy. Nature 2005, 434, 917-921.

3. Murai, J.; Huang, S.Y.; Das, B.B.; Renaud, A.; Zhang, Y.; Doroshow, J.H.; Ji, J.; Takeda, S.; Pommier, Y. Trapping of PARP1 and PARP2 by Clinical PARP Inhibitors. Cancer Res. 2012, 75, 5588-5599.

4. Fong, P.C.; Boss, D.S.; Yap, T.A.; Tutt, A.; Wu, P.; Mergui-Roelvink, M.; Mortimer, P.; Swaisland, H.; Lau, A.; O'Connor, M.J.; et al. Inhibition of poly(ADP-ribose) polymerase in tumors from BRCA mutation carriers. N. Engl. J. Med. 2009, 361, 123-134.

5. Bundred, N.; Gardovskis, J.; Jaskiewicz, J.; Eglitis, J.; Paramonov, V.; McCormack, P.; Swaisland, H.; Cavallin, M.; Parry, T.; Carmichael, J.; et al. Evaluation of the pharmacodynamics and pharmacokinetics of the PARP inhibitor olaparib: A Phase I multicentre trial in patients scheduled for elective breast cancer surgery. Investig. New Drugs 2013, 31, 949-958.

6. Gelmon, K.A.; Tischkowitz, M.; Mackay, H.; Swenerton, K.; Robidoux, A.; Tonkin, K.; Hirte, H.; Huntsman, D.; Clemons, M.; Gilks, B.; et al. Olaparib in patients with recurrent high-grade serous or poorly differentiated ovarian carcinoma or triple-negative breast cancer: A phase 2, multicentre, open-label, non-randomised study. Lancet Oncol. 2011, 12, 852-861.

7. Tutt, A.; Robson, M.; Garber, J.E.; Domchek, S.M.; Audeh, M.W.; Weitzel, J.N.; Friedlander, M.; Arun, B.; Loman, N.; Schmutzler, R.K.; et al. Oral poly(ADP-ribose) polymerase inhibitor olaparib in patients with BRCA1 or BRCA2 mutations and advanced breast cancer: A proof-ofconcept trial. Lancet 2010, 376, 235-244.

8. Chen, Y.; Zhang, L.; Hao, Q. Olaparib: A promising PARP inhibitor in ovarian cancer therapy. Arch. Gynecol. Obstet. 2013, 288, 367-374.

9. Kaye, S.B.; Lubinski, J.; Matulonis, U.; Ang, J.E.; Gourley, C.; Karlan, B.Y.; Amnon, A.; Bell-McGuinn, K.M.; Chen, L.M.; Friedlander, M.; et al. Phase II, open-label, randomized, multicenter study comparing the efficacy and safety of olaparib, a poly (ADP-ribose) polymerase inhibitor, and pegylated liposomal doxorubicin in patients with BRCA1 or BRCA2 mutations and recurrent ovarian cancer. J. Clin. Oncol. 2012, 30, 372-379.

10. Ledermann, J.; Harter, P.; Gourley, C.; Friedlander, M.; Vergote, I.; Rustin, G.; Scott, C.; Meier, W.; Shapira-Frommer, R.; Safra, T.; et al. Olaparib maintenance therapy in platinum-sensitive relapsed ovarian cancer. $N$. Engl. J. Med. 2012, 366, 1382-1392. 
11. Liu, J.F.; Tolaney, S.M.; Birrer, M.; Fleming, G.F.; Buss, M.K.; Dahlberg, S.E.; Lee, H.; Whalen, C.; Tyburski, K.; Winer, E.; et al. A Phase 1 trial of the poly(ADP-ribose) polymerase inhibitor olaparib (AZD2281) in combination with the anti-angiogenic cediranib (AZD2171) in recurrent epithelial ovarian or triple-negative breast cancer. Eur. J. Cancer 2013, 49, 2972-2978.

12. Moon, D.H.; Lee, J.M.; Noonan, A.M.; Annunziata, C.M.; Minasian, L.; Houston, N.; Hays, J.L.; Kohn, E.C. Deleterious BRCA1/2 mutation is an independent risk factor for carboplatin hypersensitivity reactions. Br. J. Cancer 2013, 109, 1072-1078.

13. Miura, K.; Sakata, K.; Someya, M.; Matsumoto, Y.; Matsumoto, H.; Takahashi, A.; Hareyama, M. The combination of olaparib and camptothecin for effective radiosensitization. Radiat. Oncol. 2012, 7, 62 .

14. Senra, J.M.; Telfer, B.A.; Cherry, K.E.; McCrudden, C.M.; Hirst, D.G.; O’Connor, M.J.; Wedge, S.R.; Stratford, I.J. Inhibition of PARP-1 by olaparib (AZD2281) increases the radiosensitivity of a lung tumor xenograft. Mol. Cancer Ther. 2011, 10, 1949-1958.

15. Yamamoto, N.; Nokihara, H.; Yamada, Y.; Goto, Y.; Tanioka, M.; Shibata, T.; Yamada, K.; Asahina, H.; Kawata, T.; Shi, X.; et al. A Phase I, dose-finding and pharmacokinetic study of olaparib (AZD2281) in Japanese patients with advanced solid tumors. Cancer Sci. 2012, 103, 504-509.

16. Khan, O.A.; Gore, M.; Lorigan, P.; Stone, J.; Greystoke, A.; Burke, W.; Carmichael, J.; Watson, A.J.; McGown, G.; Thorncroft, M.; et al. A phase I study of the safety and tolerability of olaparib (AZD2281, KU0059436) and dacarbazine in patients with advanced solid tumours. Br. J. Cancer 2011, 104, 750-755.

17. Samol, J.; Ranson, M.; Scott, E.; Macpherson, E.; Carmichael, J.; Thomas, A.; Cassidy, J. Safety and tolerability of the poly(ADP-ribose) polymerase (PARP) inhibitor, olaparib (AZD2281) in combination with topotecan for the treatment of patients with advanced solid tumors: A phase I study. Investig. New Drugs 2012, 30, 1493-1500.

18. Dean, E.; Middleton, M.R.; Pwint, T.; Swaisland, H.; Carmichael, J.; Goodege-Kunwar, P.; Ranson, M. Phase I study to assess the safety and tolerability of olaparib in combination with bevacizumab in patients with advanced solid tumours. Br. J Cancer 2012, 106, 468-474.

19. Rajan, A.; Carter, C.A.; Kelly, R.J.; Gutierrez, M.; Kummar, S.; Szabo, E.; Yancey, M.A.; Ji, J.; Mannargudi, B.; Woo, S.; et al. A phase I combination study of olaparib with cisplatin and gemcitabine in adults with solid tumors. Clin. Cancer Res. 2012, 18, 2344-2351.

20. Nijenhuis, C.M.; Lucas, L.; Rosing, H.; Schellens, J.H.M.; Beijnen, J.H. Development and validation of high-performance liquid chromatography-tandem mass spectrometry assay quantifying olaparib in human plasma. J. Chromatogr. B Anal. Technol. Biomed. Life Sci. 2013, 940, 121-125.

21. Sparidans, R.W.; Martens, I.; Valkenburg-van Iersel, L.B.; den Hartigh, J.; Schellens, J.H.; Beijnen, J.H. Liquid chromatography-tandem mass spectrometric assay for the PARP-1 inhibitor olaparib in combination with the nitrogen mustard melphalan in human plasma. J. Chromatogr. B Analy. Technol. Biomed. Life Sci. 2011, 879, 1851-1856. 
22. U.S. Food and Drug Administration. Guidance for Industry: Bioanalytical Method Validation; U.S. Food and Drug Administration: Silver Spring, MD, USA, 2001.

(C) 2014 by the authors; licensee MDPI, Basel, Switzerland. This article is an open access article distributed under the terms and conditions of the Creative Commons Attribution license (http://creativecommons.org/licenses/by/3.0/). 